\title{
Destabilization of the Mg-H System through Elastic Constraints
}

\author{
A. Baldi, ${ }^{1, *}$ M. Gonzalez-Silveira, ${ }^{1}$ V. Palmisano, ${ }^{1}$ B. Dam, ${ }^{2}$ and R. Griessen ${ }^{1}$ \\ ${ }^{1}$ Department of Physics and Astronomy, VU University Amsterdam, De Boelelaan 1081, 1081 HV Amsterdam, The Netherlands \\ ${ }^{2}$ DelftChemTech, Technical University Delft, Julianalaan 136, 2600 GA Delft, The Netherlands
}

(Received 10 March 2009; published 3 June 2009)

\begin{abstract}
We tune the thermodynamics of hydrogen absorption in $\mathrm{Mg}$ by means of elastic clamping. The loading isotherms measured by hydrogenography show that $\mathrm{Mg}$ films covered with $\mathrm{Mg}$-alloy-forming elements, such as Pd and $\mathrm{Ni}$, have hydrogen plateau pressures more than 2 orders of magnitude higher than bulk $\mathrm{Mg}$ at the same temperature. An elastic model allows us to interpret the Mg thickness dependence of the hydrogen plateau pressure. Our results suggest an alternative route for the development of new hydrogen storage materials with optimized thermodynamic properties.
\end{abstract}

DOI: 10.1103/PhysRevLett.102.226102

PACS numbers: 68.60.- $\mathrm{p}, 62.20 .-\mathrm{x}, 68.65 . \mathrm{Ac}$

Hydrogen is an attractive energy carrier for a future sustainable energy system. Compact hydrogen storage is however still a scientific and technological challenge [1]. Storage of molecular hydrogen, both gaseous and liquid, requires high pressures or very low temperatures and is thus not energy efficient. An alternative is to store atomic hydrogen in metal or complex hydrides. Storage in metal hydrides allows us, in principle, to achieve high volumetric and gravimetric densities and to reversibly operate at room temperature and atmospheric pressure. However, there are thermodynamic and kinetic limitations associated with the chemical reactions involved in hydrogen absorption and desorption.

An optimal metal-hydride system has a hydrogen equilibrium pressure of 1 bar at room temperature. Because of weight constraints the interest of the scientific community has turned to lightweight hydride-forming elements such as $\mathrm{Li}, \mathrm{B}, \mathrm{Na}, \mathrm{Mg}$, and $\mathrm{Al}$. $\mathrm{Mg}$ can store up to 7.6 mass\% of hydrogen, forming the ionic $\mathrm{MgH}_{2}$ compound, but shows poor kinetics of hydrogen absorption or desorption and a hydrogen equilibrium pressure of 1 bar at the far too high temperature of $573 \mathrm{~K}$. The absorption and desorption kinetics can be enhanced by reducing the size of the $\mathrm{Mg}$ grains via ball milling [2], hence shortening the hydrogen diffusion length, or by addition of proper catalysts [3]. Many efforts are currently dedicated to modifying the thermodynamics of the $\mathrm{Mg}-\mathrm{H}$ system in order to promote hydrogen dissociation at lower temperatures. Some destabilization has been observed upon reduction of particle size [4] and alloying of $\mathrm{Mg}$ with other elements [5,6], although this generally implies a reduction in the total hydrogen storage capacity. Theoretical works suggest the possibility to reduce the hydrogen desorption temperature in both $\mathrm{MgH}_{2}$ nanoparticles with grain size smaller than $\sim 1.3 \mathrm{~nm}$ [7] and in $\mathrm{MgH}_{2}$ layers with thicknesses below 10 unit cells [8].

In this Letter we show that the thermodynamic stability of the $\mathrm{Mg}-\mathrm{H}$ system can be drastically modified via elastic constraints. This is a direct consequence of the long-range
$\mathrm{H}-\mathrm{H}$ interaction in metals $[9,10]$. In a metal with elastically free surfaces two hydrogen atoms feel an effective attractive interaction, while in a sample clamped on all sides the interaction becomes repulsive. Clamping can thus be used to tune the equilibrium hydrogen pressure to the levels required for specific applications. To prove the validity of our approach we prepared two series of capped $\mathrm{Mg}$ thin films: (1) to explore the role of chemical binding we deposited $\mathrm{Mg}$ thin films capped with various transition metals, differing in their affinities towards magnesium and in their hydrogen solubilities: $\mathrm{Ni}, \mathrm{Pd}, \mathrm{Ti}, \mathrm{Nb}$, and $\mathrm{V}$; (2) in order to study the thickness dependence of the elastic effect we prepared a series of Pd-capped $\mathrm{Mg}$ films of different thicknesses. The effect of clamping is strongly dependent on the chemical nature of the transition metal used as a cap layer and will be interpreted with a simple elastic model. By choosing the appropriate sample geometry we are able to increase the hydrogen plateau pressure more than 200 times with respect to bulk Mg. Hydrogen absorption in our samples is measured by means of optical spectroscopy and hydrogenography [6], a novel technique that allows to measure pressure-optical transmission isotherms (PTI's). In a PTI the amount of light transmitted by a thin film is measured as a function of increasing pressure at constant temperature: when the metallic $\mathrm{Mg}$ films load with hydrogen, the $\mathrm{Mg}-\mathrm{MgH}_{2}$ metal-insulator transition leads to an abrupt increase in the amount of transmitted light. From the Beer-Lambert law, $\ln \left(T / T_{M}\right) \propto c_{\mathrm{H}} d$, the logarithm of the optical transmission, $T$, normalized for the transmission of the film in its metallic state, $T_{M}$, is proportional to the hydrogen concentration in the film, $c_{\mathrm{H}}$, and to the film thickness, $d$. Measuring PTI's is therefore in all respects equivalent to measuring standard pressurecomposition isotherms [11]. The films used in this study are deposited in a UHV chamber $\left(p<10^{-6} \mathrm{~Pa}\right.$ ) by a rf-dc magnetron sputtering on glass and quartz substrates. All films are covered with Pd to prevent oxidation and catalyze hydrogen absorption. Optical reflection spectra are measured in a PerkinElmer Lambda 900 diffraction grating 


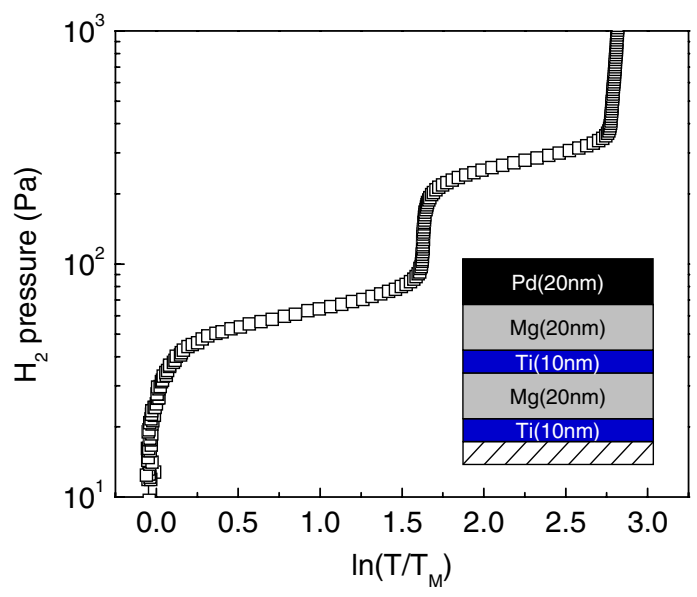

FIG. 1 (color online). Loading PTI measured at $333 \mathrm{~K}$ for a $2 \times[\mathrm{Ti}(10 \mathrm{~nm}) \mathrm{Mg}(20 \mathrm{~nm})] \operatorname{Pd}(10 \mathrm{~nm})$ sample deposited on glass. The inset is a cartoon of the sample geometry: glass-substrate $/ \mathrm{Ti} / \mathrm{Mg} / \mathrm{Ti} / \mathrm{Mg} / \mathrm{Pd}$.

spectrometer, with energy range between 0.5 and $6.5 \mathrm{eV}$, during (de)hydrogenation at room temperature and $\mathrm{H}_{2}$ pressures up to $10^{5} \mathrm{~Pa}$. PTI's are recorded in an optical cell that allows the variation of the hydrogen pressure from $10^{-1}$ to $10^{6} \mathrm{~Pa}$ at constant temperatures, between 313 and $573 \mathrm{~K}$. Further details on the hydrogenography experimental setup can be found in Ref. [6]. In Fig. 1 the PTI of a $2 \times[\operatorname{Ti}(10 \mathrm{~nm}) \operatorname{Mg}(20 \mathrm{~nm})]$ film covered with $10 \mathrm{~nm}$ of $\mathrm{Pd}$ is shown. In the pressure and temperature ranges explored $\mathrm{Pd}$ and $\mathrm{Ni}$ do not load with hydrogen. $\mathrm{Ti}, \mathrm{Nb}$, and $\mathrm{V}$ layers on the contrary load at the beginning of the pressure ramp but, due to the metallic nature of their hydrides, produce very small optical effects compared to $\mathrm{Mg}$. The observed plateaus are therefore only due to the formation of $\mathrm{MgH}_{2}$ upon hydrogen absorption. The isotherm shown in Fig. 1 exhibits two clear and distinct plateaus of similar width. Since the width of the plateau is proportional to the thickness of the material, the two plateaus must originate from the two distinct Mg layers. Furthermore, to obtain such a well defined double plateau we have to assume that the two $\mathrm{Mg}$ layers are elastically disconnected [12] and have different site energies for hydrogen absorption. To prove these hypotheses we measure the optical reflection of the $2 \times$ $[\mathrm{Ti}(10 \mathrm{~nm}) \operatorname{Mg}(20 \mathrm{~nm})] \operatorname{Pd}(20 \mathrm{~nm})$ sample deposited on quartz, while slowly increasing the hydrogen pressure. Optical spectra are measured through the transparent quartz substrate at near-normal incidence. In Fig. 2(a) the measured spectra are shown for three different steps of loading: (1) as-deposited metallic sample, (2) at intermediate loading, and (3) fully loaded sample. The calculated spectra shown in Fig. 2(b) are generated with SCOUT [13] by taking into account the volume expansion occurring upon hydrogenation of $\mathrm{Mg}$ and $\mathrm{Ti}$ and including a rough interface between the quartz substrate and the first Ti layer. In the simulation the intermediate step is due to the complete loading of one of the two $\mathrm{Mg}$ layers while the other

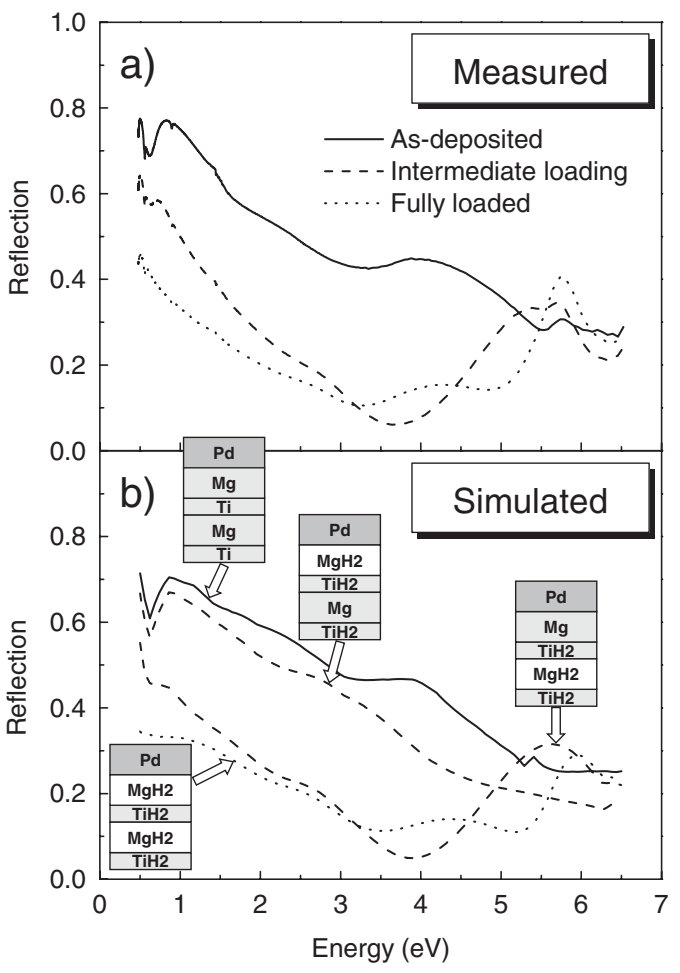

FIG. 2. Measured (a) and simulated (b) optical reflection measured through the quartz substrate for a $2 \times$ $[\mathrm{Ti}(10 \mathrm{~nm}) \mathrm{Mg}(20 \mathrm{~nm})]$ film covered with $20 \mathrm{~nm}$ of Pd at different stages of loading. In the simulations two possible states are taken into account for the intermediate loading: the "loading from top" and "loading from bottom." The dielectric functions of $\mathrm{Ti}, \mathrm{Mg}, \mathrm{TiH}_{2}, \mathrm{MgH}_{2}$, and $\mathrm{Pd}$ used in the simulations are taken from Refs. [21-24,21], respectively.

remains metallic. Comparison with the experimental spectra shows unambiguously that the $\mathrm{Mg}$ layer sandwiched between two Ti layers absorbs hydrogen at a lower pressure than the one capped with Pd. This is rather counterintuitive since the "Ti-sandwiched Mg" lies on the bottom of the sample and hydrogen has to diffuse through the top $\mathrm{Mg}$ layer, which remains in the diluted $\alpha$ phase, in order to reach the bottom one. For this mechanism to take place, the two Mg layers have to differ with respect to their thermodynamic properties, and they must be elastically disconnected by the presence of the Ti layer in between. This remarkable behavior is the result of the following $\mathrm{H}$ loading sequence. Already at very low $\mathrm{H}_{2}$ pressures Ti forms $\mathrm{TiH}_{2}$. The consequent $25 \%$ volume expansion, together with the fact that $\mathrm{Mg}$ and $\mathrm{Ti}$ are immiscible, leads to reduced strains at the $\mathrm{Mg} / \mathrm{TiH}_{2}$ interfaces. The Tisandwiched $\mathrm{Mg}$ layer is then effectively elastically disconnected from the surrounding. However, since Pd alloys with $\mathrm{Mg}$ and loads at higher pressures, this reasoning is not applicable to the Ti-Mg-Pd block. When the top $\mathrm{Mg}$ layer expands upon $\mathrm{H}$ absorption, it feels the Pd elastic constraint, resulting in a higher hydride formation enthalpy and consequently in a higher plateau pressure. To demon- 


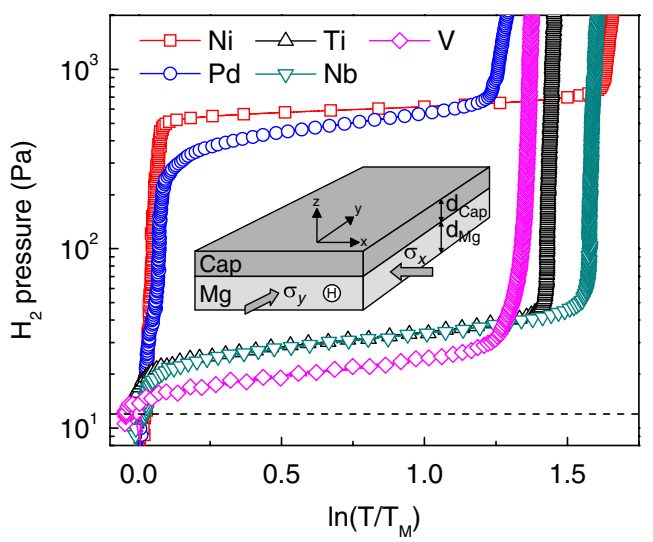

FIG. 3 (color online). Effect of cap layer: PTI's measured at $333 \mathrm{~K}$ for $\operatorname{Ti}(10 \mathrm{~nm}) \operatorname{Mg}(20 \mathrm{~nm}) X(10 \mathrm{~nm}) \operatorname{Pd}(10 \mathrm{~nm})$ samples deposited on glass with $X=\mathrm{Ni}, \mathrm{Pd}, \mathrm{Ti}, \mathrm{Nb}$, and $\mathrm{V}$. The dashed line is the pressure at which coexistence of $\alpha$ and $\beta$ phases begins to appear upon hydrogen absorption in bulk Mg [15]. In the inset: schematic visualization of the elastic model used to interpret the data.

strate the validity of this interpretation we considered two series of samples: (a) Mg thin films capped with different transition metals, and (b) Pd-capped $\mathrm{Mg}$ thin film with different thicknesses. Figure 3 shows the PTI's for 5 different samples with the following geometry: $\operatorname{Ti}(10 \mathrm{~nm}) \operatorname{Mg}(20 \mathrm{~nm}) X(10 \mathrm{~nm}) \operatorname{Pd}(10 \mathrm{~nm})$ with $X=\mathrm{Ni}$, $\mathrm{Pd}, \mathrm{Ti}, \mathrm{Nb}$, and $\mathrm{V}$. Because of the presence of a Ti layer between $\mathrm{Mg}$ and the substrate we can assume that the only clamping effect is due to the top layer. Clearly two behaviors are distinguishable: (1) Mg-alloy-forming elements such as $\mathrm{Ni}$ and Pd have a strong effect on the thermodynamic properties of $\mathrm{Mg}$, leading to a much higher plateau pressure than the one measured for $\mathrm{Mg}$ films of equal thickness capped with $\mathrm{Ti}, \mathrm{Nb}$, and $\mathrm{V}$; (2) the latter elements, which are immiscible with $\mathrm{Mg}$, effectively behave like "scissors" and lead to "quasifree" Mg layers with elastically disconnected interfaces. Assuming that the origin of the destabilization observed is only elastic in nature, we can build a simple model consisting of two layers-a $\mathrm{Mg}$ and a cap layer-glued to each other as shown in the inset in Fig. 3: hydrogen atoms absorbed by the Mg layer isotropically expand the lattice of the metallic host, while the $\mathrm{Mg}$ feels a compressive stress in the $x$ and $y$ directions $\left(\sigma_{x}\right.$ and $\left.\sigma_{y}\right)$ due to the cap layer. In the $z$ direction there is no stress built up by the clamping, but we have to take into account the strain along the $z$ direction due to the Poisson's ratio, $\nu$. The two layers are in vacuum, and no effect of the substrate is taken into account, basically assuming that the bottom Ti layer is a perfect scissor. By taking into account only elastic constraints we can calculate the effective volume expansion of the $\mathrm{Mg}$ layer upon hydrogen absorption. Furthermore, the enthalpy of hydride formation has a simple volume dependence through the bulk modulus, $B$ [14]: $d \Delta H / d \ln V=$
$-B_{\mathrm{Mg}} V_{\mathrm{H}}$, where $V_{\mathrm{H}}$ is the partial molar volume of hydrogen in $\mathrm{Mg}$. We can then derive an expression for the plateau pressure of the capped magnesium layer, $\mathrm{Mg}^{*}$, with respect to free bulk magnesium, $\mathrm{Mg}^{0}$ :

$$
\ln \left(\frac{p^{*}}{p^{0}}\right)=\frac{E_{\mathrm{Mg}}}{\tilde{E}}\left[1-\nu_{\mathrm{Mg}}+\left(1-\nu_{\mathrm{Cap}}\right) \frac{E_{\mathrm{Mg}}}{E_{\mathrm{Cap}}} \frac{d_{\mathrm{Mg}}}{d_{\mathrm{Cap}}}\right]^{-1},
$$

where $\tilde{E}=\frac{9}{4} R T V_{\mathrm{Mg}} / V_{\mathrm{H}}^{2}, V_{\mathrm{Mg}}$ is the molar volume of $\mathrm{Mg}$, and $E=3 B(1-2 \nu)$ is the Young's modulus. The equation can also be rewritten as $\left[\ln \left(p^{*} / p^{0}\right)\right]^{-1}=I+S d_{\mathrm{Mg}}$ with

$$
I=\frac{\tilde{E}}{E_{\mathrm{Mg}}}\left(1-\nu_{\mathrm{Mg}}\right), \quad S=\frac{\tilde{E}}{E_{\mathrm{Cap}}}\left(1-\nu_{\mathrm{Cap}}\right) \frac{1}{d_{\text {Cap }}} .
$$

According to the model the equilibrium pressure for the capped $\mathrm{Mg}$ has a straightforward dependence on the thickness of the $\mathrm{Mg}$ layer, $d_{\mathrm{Mg}}$. Figure 4 shows the PTI's for 5 samples with different $\mathrm{Mg}$ thicknesses: $\operatorname{Ti}(10 \mathrm{~nm}) \operatorname{Mg}(z \mathrm{~nm}) \operatorname{Pd}(40 \mathrm{~nm})$, with $z=10,15,20,30$, and $40 \mathrm{~nm}$. Two effects are clearly visible: (a) the width of the plateau, $w$, is proportional to $d_{\mathrm{Mg}}$ as expected from the Beer-Lambert law; (b) the plateau pressure decreases with increasing $\mathrm{Mg}$ thickness as predicted by the model. A $10 \mathrm{~nm}$ thick $\mathrm{Mg}$ film has an equilibrium hydrogen pressure of $2.8 \times 10^{3} \mathrm{~Pa}$ at $333 \mathrm{~K}$, more than 200 times higher than bulk $\mathrm{Mg}$ at the same temperature $\left(p_{333 \mathrm{~K}}^{0}=12 \mathrm{~Pa}[15]\right)$. The plateau width is measured by dividing each isotherm into three regions - the solid solution ( $\alpha$ phase) before the plateau, the coexistence region ( $\alpha+\beta$ phases), and the fully hydrogenated region ( $\beta$ phase) after the plateau - and fitting each part with a straight line; the width is then given by the distance, in units of $\ln \left(T / T_{M}\right)$, between the intersection points. With the same geometrical construction we obtain the equilibrium pressure, as the pressure in the

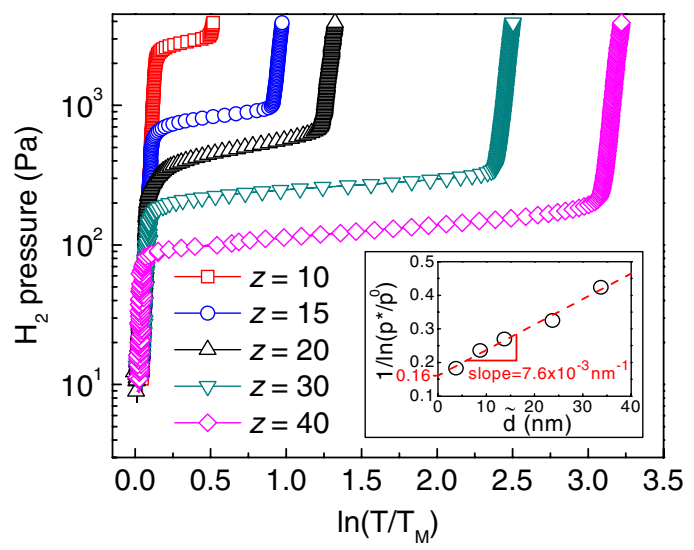

FIG. 4 (color online). Effect of Mg thickness: PTI's measured at $333 \mathrm{~K}$ for $\mathrm{Ti}(10 \mathrm{~nm}) \mathrm{Mg}(z \mathrm{~nm}) \operatorname{Pd}(40 \mathrm{~nm})$ samples deposited on glass with $z=10,15,20,30$, and $40 \mathrm{~nm}$. In the inset: $\mathrm{Mg}$ thickness dependence of the equilibrium pressure. The dashed line is a linear fit to the points. 
middle of the plateau. Ideally one would expect $w \rightarrow 0$ for $d_{\mathrm{Mg}} \rightarrow 0$; however, although the plateau width is found to be proportional to the $\mathrm{Mg}$ thickness, a linear fit shows a relation of the type $w=A\left(d_{\mathrm{Mg}}-d_{0}\right)$, with $d_{0}=6 \mathrm{~nm}$. The presence of an intercept indicates that the total amount of $\mathrm{Mg}$ undergoing a phase transition to $\mathrm{MgH}_{2}$ is thinner than expected and that we can assume for all samples that $\sim 6 \mathrm{~nm}$ of $\mathrm{Mg}$ are "lost" due to alloying with the top Pd layer. In the comparison with the model we will therefore take into account an effective Mg thickness $\tilde{d}=d_{\mathrm{Mg}}-d_{0}$. The formation of an interfacial $\mathrm{Mg}$-Pd alloy is directly responsible for the strong clamping effect observed and is consistent with the difference in width observed in the two plateaus of Fig. 1, corresponding exactly to $6 \mathrm{~nm}$. The inset in Fig. 4 shows the thickness dependence of the plateau pressure of $\mathrm{Pd}$-capped $\mathrm{Mg}$ layers: plotting $\left[\ln \left(p^{*} / p^{0}\right)\right]^{-1}$ versus the effective $\mathrm{Mg}$ thickness we find a linear dependence as predicted by the model, with intercept $I=0.16$ and slope $S=7.6 \times 10^{-3} \mathrm{~nm}^{-1}$. Substituting into Eq. (2) the literature values for the structural parameters of $\mathrm{Mg}, \mathrm{Pd}$, and $\mathrm{H} \quad\left(V_{\mathrm{Mg}}=\right.$ $13.97 \mathrm{~cm}^{3} / \mathrm{mol}, V_{\mathrm{H}}=2.24 \mathrm{~cm}^{3} / \mathrm{mol}$ [16], $\nu_{\mathrm{Mg}}=0.29$, $\left.\nu_{\mathrm{Pd}}=0.39, E_{\mathrm{Mg}}=45.2 \mathrm{GPa}, E_{\mathrm{Pd}}=126 \mathrm{GPa}\right)$ and taking $T=333 \mathrm{~K}$ and $d_{\mathrm{Pd}}=40 \mathrm{~nm}$, we obtain the theoretical values $I_{\text {th }}=0.27$ and $S_{\text {th }}=2.1 \times 10^{-3} \mathrm{~nm}^{-1}$. The agreement between experiment and theory is surprising when considering the strong approximations made in the model: no substrate is taken into account, the only interaction is elastic, hence neglecting surface energy contributions, and the layers are assumed to behave as perfect elastic bodies. The rather large $S$ value obtained from the fit can be explained considering the alloying effect taking place at the $\mathrm{Mg} / \mathrm{Pd}$ interface and assuming that only a fraction of $\sim 10$ out of $40 \mathrm{~nm}$ of Pd has a significant clamping effect on the $\mathrm{Mg}$ underneath. The discrepancy in the $I$ values could reflect the fact that we consider the Young's modulus of metallic Mg: taking into account $\mathrm{MgH}_{2}$, which is stiffer than $\mathrm{Mg}$, would lead to a closer agreement between experiment and theory. The elastic model developed for a double layer can be easily extended to three-dimensional $\mathrm{Mg}$ nanoparticles embedded in a skin of hard material. Assuming Mg particles of $10 \mathrm{~nm}$ of diameter and a skin of $2 \mathrm{~nm}$ thickness, the model predicts an increase in hydrogen equilibrium pressure of more than 2000 times with respect to bulk $\mathrm{Mg}$ at the same temperature. The effect of clamping described in the present work is not only important in the perspective of hydrogen storage applications but it is crucial in understanding the thermodynamics of all hydrogenabsorbing capped thin films, from switchable mirrors [17] to electrode materials for batteries [18], hydrogen detectors [19], multilayers, and superlattices [20].

In conclusion we have shown the possibility to tailor the thermodynamics of a metal-hydrogen system by means of elastic constraints. Thin films of $\mathrm{Mg}$ are used as a model metal-hydrogen system. Elements immiscible with $\mathrm{Mg}$ behave like scissors, while $\mathrm{Mg}$-alloy-forming elements exert a clamping effect that leads to huge increases in hydrogen equilibrium pressures. Clamping arises as a consequence of alloying between $\mathrm{Mg}$ and the top layer, and its effects can be understood on the basis of a simple elastic model. The possibility to tune the thermodynamics of a metal-hydrogen system by elastic means offers attractive new possibilities for compact hydrogen storage.

This work is supported by the Technologiestichting STW, the Nederlandse Organisatie voor Wetenschappelijk Onderzoek (NWO) through the Sustainable Hydrogen Programme of Advanced Chemical Technologies for Sustainability (ACTS), and the Marie Curie Actions through the project Complex Solid State Reactions for Energy Efficient Hydrogen Storage (COSY:RTN035366).

*abaldi@few.vu.nl

[1] L. Schlapbach and A. Züttel, Nature (London) 414, 353 (2001).

[2] A. Zaluska et al., J. Alloys Compd. 288, 217 (1999).

[3] W. Oelerich et al., J. Alloys Compd. 315, 237 (2001).

[4] R. Varin et al., J. Alloys Compd. 424, 356 (2006).

[5] J. J. Vajo et al., J. Phys. Chem. B 108, 13977 (2004).

[6] R. Gremaud et al., Adv. Mater. 19, 2813 (2007).

[7] R. Wagemans et al., J. Am. Chem. Soc. 127, 16675 (2005).

[8] J. Liang, Appl. Phys. A 80, 173178 (2005).

[9] H. Zabel and H. Peisl, Phys. Rev. Lett. 42, 511 (1979).

[10] H. Wagner and H. Horner, Adv. Phys. 23, 587 (1974).

[11] R. Gremaud et al., Appl. Phys. Lett. 91, 231916 (2007).

[12] H. Peisl, Topics in Applied Physics: Hydrogen in Metals I, edited by G. Alefeld and J. Völkl (Springer, Berlin, 1978).

[13] W. Theiss, SCOUT Thin Film Analysis Software Handbook, Hard and Software (Aachen, 2000), www.mtheiss.com.

[14] R. Griessen and R. Feenstra, J. Phys. F 15, 1013 (1985).

[15] A. Krozer and B. Kasemo, J. Less-Common Met. 160, 323 (1990).

[16] Assuming 32\% volume expansion upon hydrogenation of $\mathrm{Mg}$.

[17] J. N. Huiberts et al., Nature (London) 380, 231 (1996).

[18] R. A. H. Niessen and P. H. L. Notten, Electrochem. SolidState Lett. 8, A534 (2005).

[19] M. Slaman et al., Sensors and Actuators B: Chemical 123, 538 (2007).

[20] G. Andersson et al., Phys. Rev. B 55, 1774 (1997).

[21] E. D. Palik, Handbook of Optical Constants of Solids (Academic Press, San Diego, 1998).

[22] R. Machorro et al., Thin Solid Films 269, 1 (1995).

[23] D. M. Borsa et al., Phys. Rev. B 75, 205408 (2007).

[24] J. Isidorsson et al., Phys. Rev. B 68, 115112 (2003). 